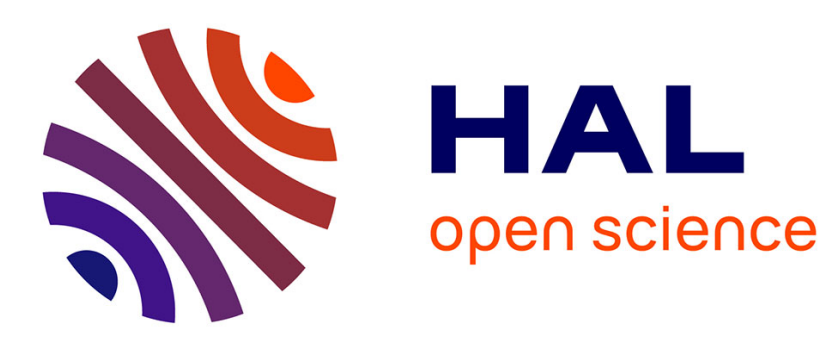

\title{
Reasoning with partially ordered information in a possibilistic logic framework
}

Salem Benferhat, Sylvain Lagrue, Odile Papini

\section{To cite this version:}

Salem Benferhat, Sylvain Lagrue, Odile Papini. Reasoning with partially ordered information in a possibilistic logic framework. Fuzzy Sets and Systems, 2004, 144 (1), pp.25-41. 10.1016/j.fss.2003.10.012 . hal-01479591

\section{HAL Id: hal-01479591 https://hal-amu.archives-ouvertes.fr/hal-01479591}

Submitted on 10 Feb 2020

HAL is a multi-disciplinary open access archive for the deposit and dissemination of scientific research documents, whether they are published or not. The documents may come from teaching and research institutions in France or abroad, or from public or private research centers.
L'archive ouverte pluridisciplinaire HAL, est destinée au dépôt et à la diffusion de documents scientifiques de niveau recherche, publiés ou non, émanant des établissements d'enseignement et de recherche français ou étrangers, des laboratoires publics ou privés. 


\section{Reasoning with Partially Ordered Information in a Possibilistic Logic Framework}

\author{
Salem Benferhat \\ IRIT \\ Univerité Paul Sabatier, \\ 118, route de Narbonne, \\ 31062 Toulouse Cedex 4 \\ benferhat@irit.fr
}

\author{
Sylvain Lagrue \\ PRAXITEC \\ 115 , r St Jacques \\ 13006 Marseille \\ lagrue@irit.fr
}

\author{
Odile Papini \\ SIS \\ Université de Toulon et du Var, \\ av. de l'université BP 132, \\ 83957 La Garde, cedex \\ papini@univ-tln.fr
}

\begin{abstract}
Possibilistic logic offers a natural qualitative framework for handling uncertain information. This paper discusses its extension to dealing with partially ordered knowledge. We show that there are two different ways to define possibilistic logic machinery which both extend the standard one.
\end{abstract}

Keywords: possibilistic logic, partial preorders.

\section{Introduction}

In many applications, the reliability relation associated with available information is only partially defined, while most of existing uncertainty frameworks deal with totally ordered knowledge. Partial orders offer more flexibility than total pre-orders to represent incomplete knowledge. Moreover, they avoid to compare unrelated pieces of information.

The need of extension of uncertainty frameworks to partial orders is even crucial if we consider the dynamic of knowledge. Namely, even if the available information is totally ordered, then it may happen, when using updating operators [1], that incorporating a new piece of information leads to a partially ordered knowledge.
Another situation is when we merge multiple sources information. Indeed the application of some merging technique, like the one based on preferred sub-theories [2], can also result in a set of partially ordered pieces of information.

This paper proposes an extension of basic notions of possibilistic logic [3], when pieces of information are only partially ordered. Namely, instead of associating with formulas or interpretations numbers in $[0,1]$, we will associate elements in some partially ordered set.

Two definitions of possibilistic inference, which both extend the one used in possibilistic logic, are presented.

The paper is organised as follows. Section 2 gives some definitions on partial pre-orders. Section 3 proposes an extension of the semantic of possibilistic logic. Section 4 provides two different methods to compactly encode a partial pre-order on interpretations by means of a partial pre-order on formulas. We then propose a syntactic inference, which extends the possibilistic one.

\section{Partial pre-orders: definitions and notations}

A partial pre-order $\preceq$ on a set $A$ is a reflexive ( $a \preceq a$ ) and a transitive binary relation (if $a \preceq b$ and $b \preceq c$ then $a \preceq c$ ). 
In this paper, $a \preceq b$ means that $a$ is preferred to $b$.

We define the corresponding strict partial order as $a \prec b$ iff $a \preceq b$ holds and $b \preceq a$ does not hold. The equality $=$ is defined by $a=b$ iff $a \preceq b$ and $b \preceq a$.

We lastly define incomparability $\sim$ as $a \sim b$ if and only if neither $a \preceq b$ nor $b \preceq a$ holds. $a \sim b$ means that neither $a$ is preferred to $b$, nor the converse.

The set of minimal elements of $A$, denoted by $\operatorname{Min}(A, \preceq))$, is defined as follows: $\operatorname{Min}(A, \preceq)=\{a \in A: \nexists b \in A, b \prec a\}$.

From a partial pre-order on elements to a partial pre-order on subsets of elements

Given a partial pre-order $\preceq$ on a set $A$, we need to define, for the purpose of this paper, a partial pre-order between subsets of $A$. Roughly speaking, a subset $X$ is preferred to another subset $Y$, if the preferred elements in $X$ (represented by $\operatorname{Min}(X, \preceq)$ ) is better than the preferred elements of $Y$ (represented by $\operatorname{Min}(Y, \preceq)$ ).

Following Halpern [5] [6], there are two meaningful ways to compare subsets of $A$.

Definition 1 Let $\preceq$ be a partial pre-order on $A$ and $X, Y \in A . \quad X \preceq_{1} Y$ iff $\forall y \in Y$, $\exists x \in X$ such that $x \preceq y$.

This definition means that a subset $X$ is preferred to another subset $Y$ if for each element $y$ in $Y$, there exists at least one element in $X$, which is preferred to $y$.

The following gives a stronger definition.

Definition 2 Let a partial pre-order $\preceq$ on $A$ and $X, Y \in A . X \preceq_{2} Y$ iff $\exists x \in X$ such that $\forall y \in Y: x \preceq y$.

With this definition, we consider that a subset $X$ is preferred to another subset $Y$ when there exists at least one element in $X$ which is preferred to all elements in $Y$.
The definition of $\preceq_{2}$ implies the definition of $\preceq_{1}$, more formally:

Proposition $1 \forall X, Y \in A$, if $X \preceq_{2} Y$ then $X \preceq_{1} Y$.

The converse of this proposition does not hold, as it is shown by the following counterexample.

Counterexample 1 Let $A=$ $\left\{x_{1}, x_{2}, y_{1}, y_{2}\right\}$ and the partial pre-order $\preceq$ on $A$ defined by:

$$
\left\{\begin{array}{l}
x_{1} \preceq y_{1} \\
x_{2} \preceq y_{2}
\end{array}\right.
$$

Let $X$ and $Y$ be two subsets of $A$ such that $X=\left\{x_{1}, x_{2}\right\}$ and $Y=\left\{y_{1}, y_{2}\right\}$. We have $X \preceq_{1} Y$, indeed $x_{1}$ is preferred to $y_{1}$ and $x_{2}$ is preferred to $y_{2}$. But $X \preceq_{2} Y$ does not hold: there exists no element in $X$ which is preferred to all elements in $Y$.

The following proposition shows that, in the case where $\preceq$ is a total pre-order then the two definitions of $\preceq_{1}$ and $\preceq_{2}$ are equivalent.

Proposition 2 Let $\preceq$ be a total pre-order. Then: $\forall X, Y \in A, X \preceq_{1} Y$ iff $X \preceq_{2} Y$.

\section{A semantic extension for possibilistic logic}

This section provides an extension of the semantics of the possibilistic logic [3] to partial pre-orders.

Let $\mathcal{L}$ be a propositional language. We denote by $\mathcal{W}$ the set of interpretations of $\mathcal{L}$. Let $\varphi$ be a formula, $\operatorname{Mod}(\varphi)$ denotes the set of classical models of $\varphi$.

We recall that the basic element of the semantics of possibilistic logic is the notion of possibility distribution [4], denoted by $\pi$, which is a function from $\mathcal{W}$ to $[0,1] . \pi(\omega)$ evaluates to what extent $\omega$ is compatible, or consistent, with our available knowledge. 
$\pi(\omega)=0$ means that $\omega$ is impossible, while $\pi(\omega)=1$ means that $\omega$ is totally possible. If there exists an interpretation $\omega$ such that $\pi(\omega)=1, \pi$ is said to be normalized, or consistent.

The counterpart of a possibility distribution is a partial pre-order $\preceq_{\mathcal{W}}$ on $\mathcal{W}$. $\omega \preceq_{\mathcal{W}} \omega^{\prime}$ means that $\omega$ is preferred (or more plausible) than $\omega^{\prime}$.

Note that contrary to possibility distribution, we can not represent totally possible interpretations and totally impossible interpretations. We can only represent the most preferred interpretations and the least preferred interpretations. Moreover, like in Spohn Ordinal function (OCF) [7], all interpretations are considered as somewhat possible.

We now define an extension of the possibilistic semantic inference. A formula $\varphi$ is a plausible conclusion, given the available knowledge encoded by $\preceq_{\mathcal{W}}$, if it is satisfied by all preferred interpretations for $\preceq \mathcal{W}$. More formally:

Definition 3 Let $\preceq_{\mathcal{W}}$ be a partial pre-order on $\mathcal{W}$. A formula $\varphi$ is inferred, denoted $(\mathcal{W}, \preceq \mathcal{W}) \models \varphi$, if and only if $\operatorname{Min}\left(\mathcal{W}, \preceq_{\mathcal{W}}\right.$ )$\in \operatorname{Mod}(\varphi)$.

Let us illustrate this definition by the following example.

Example 1 Let $a$ and $b$ be two propositional variables. Let $\mathcal{W}=\left\{\omega_{0}, \omega_{1}, \omega_{2}, \omega_{3}\right\}$ be the set of interpretations such that $\omega_{0}=$ $\{\neg a, \neg b\}, \omega_{1}=\{\neg a, b\}, \omega_{2}=\{a, \neg b\}$ and $\omega_{3}=\{a, b\}$. Let $\preceq_{\mathcal{W}}$ be a partial pre-order on $\mathcal{W}$ such that:

$$
\left\{\begin{array}{l}
\omega_{0}=\mathcal{W} \omega_{1} \\
\omega_{2} \preceq \mathcal{W} \omega_{0} \\
\omega_{3} \preceq \mathcal{W} \omega_{0}
\end{array}\right.
$$

Note that $\omega_{2} \sim_{\mathcal{W}} \omega_{3}$ and $\operatorname{Min}\left(\mathcal{W}, \preceq_{\mathcal{W}}\right)=$ $\left\{\omega_{2}, \omega_{3}\right\}$.

We are interested to know if $a \vee b$ can be inferred from $(\mathcal{W}, \preceq \mathcal{W})$.

We have $\operatorname{Mod}(a \vee b)=\left\{\omega_{1}, \omega_{2}, \omega_{3}\right\}$. Which means that $\operatorname{Min}(\mathcal{W}, \preceq \mathcal{W}) \subseteq \operatorname{Mod}(a \vee b)$.

Therefore we have $(\mathcal{W}, \preceq \mathcal{W}) \models a \vee b$.

This inference is an extension of the one used in possibilistic logic. Namely, let $\pi$ be a normalized possibility distribution. Recall that a formula $\varphi$ is inferred from $\pi$, denoted by $\pi \models \varphi$, if each interpretation $\omega$ such that $\pi(\omega)=1$ satisfies $\varphi$.

Let $\leq_{\pi}$ be a partial pre-order associated with a possibility distribution $\pi$ in the following way: $\omega \leq_{\pi} \omega^{\prime \prime}$ iff $\pi\left(\omega^{\prime}\right) \leq \pi(\omega)$.

It can be checked that:

$\pi \models \varphi$ iff $\left(\mathcal{W}, \preceq_{\mathcal{W}}\right) \models \varphi$.

\section{A syntactic extension for possibilistic logic}

This section provides two syntactic counterparts to the semantic inference introduced in the above section. We first show how to compactly encode a partial pre-order on interpretations with a partial pre-order, denoted by $\preceq_{K}$, on a set of propositional formulas, denoted by $K$. We then build a set of consistent subsets of $K$, which will be used to define a syntactic inference.

\section{From partial pre-orders on formulas to} partial pre-orders on interpretations

In possibilistic logic, uncertain information are represented by means of a possibilistic knowledge base, which is a set of weighted formulas of the form $\Sigma=\left\{\left(\varphi_{i}, a_{i}\right): i=\right.$ $1, \ldots, n\}$ where $\varphi_{i}$ is a propositional formula, and $a_{i} \in[0,1]$. The real number $a_{i}$ represents a lower bound of certainty degree of the formula $\varphi_{i}$.

We propose to represent compactly partially ordered information by a couple $\left(K, \preceq_{K}\right)$, where $K$ represents a set of formulas and $\preceq_{K}$ a partial pre-order on this set. $\left(K, \preceq_{K}\right)$ will be called partially ordered knowledge base. 
Given two formulas $\varphi$ and $\varphi^{\prime} \in K, \varphi \preceq_{K} \varphi^{\prime}$ means that $\varphi$ is preferred to $\varphi^{\prime}$. Note that, in general, $K$ is neither deductively closed, nor required to be consistent.

From $\left(K, \preceq_{K}\right)$, we can generate two possible pre-orders on interpretations, depending if we use Definition 1 or Definition 2.

Let us denote by $\lceil\omega, K\rceil$ the set of formulas in $K$ falsified by $\omega$. We have:

Definition 4 Let $\left(K, \preceq_{K}\right)$ be a partially ordered knowledge base, then for $i=1,2$ we have $\omega \preceq_{\mathcal{W}, i} \omega^{\prime}$ if $\left\lceil\omega^{\prime}, K\right\rceil \preceq_{i}\lceil\omega, K\rceil$, where $\preceq_{i}$ is given in Definition 1 for $i=1$, and in Definition 2 if $i=2$.

Roughly speaking, $\omega$ is preferred to $\omega^{\prime}$ if the preferred elements in $\left(K, \preceq_{K}\right)$ falsified by $\omega^{\prime}$ are better than preferred formulas falsified by $\omega$.

Example 2 Let be $K=\{a, \neg b, b\}$ the set of formulas and $\preceq_{K}$ such that:

$$
\left\{\begin{array}{l}
a \preceq_{K} b \\
a \preceq_{K} \neg b
\end{array}\right.
$$

Note that $K$ is inconsistent. Moreover, we have $b \sim \neg b$. We recall that $\omega_{0}=\{\neg a, \neg b\}$, $\omega_{1}=\{\neg a, b\}, \omega_{2}=\{a, \neg b\}$ and $\omega_{3}=\{a, b\}$.

Using the definition of $\preceq_{1}$, we can show that $\omega_{2} \preceq \mathcal{W}, 1 \quad \omega_{0}$. Indeed $\left\lceil\omega_{2}, K\right\rceil=\{b\}$ and $\left\lceil\omega_{0}, K\right\rceil=\{a, b\}$.

Moreover we have: $b \preceq_{K} b$ and $a \preceq_{K} b$, hence $\{a, b\} \preceq_{1}\{b\}$, which implies $\omega_{2} \preceq_{\mathcal{W}, 1}$ $\omega_{0}$.

With similar computations, we obtain:

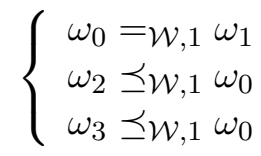

Note that this leads to the same than the partial pre-order of Example 1.

In this particular case, we can check that $\preceq_{\mathcal{W}, 1}=\preceq_{\mathcal{W}, 2}$.
The following shows that the pre-order induced on interpretations from a partially ordered knowledge base is an extension of the one use in possibilistic logic. More precisely, let $\Sigma=\left\{\left(\varphi_{i}, a_{i}\right): i=1 \ldots n\right\}$ be a possibilistic knowledge base. Recall that in possibilistic logic the possibility distribution associated with $\Sigma$ is defined by [3]:

$\pi_{\Sigma}=\left\{\begin{array}{c}1 \text { if } \forall\left(\varphi_{i}, a_{i}\right) \in \Sigma, \omega \models \varphi_{i} \\ 1-\operatorname{Max}\left\{a_{i}:\left(\varphi_{i}, a_{i}\right) \in \Sigma, \omega \not \models \varphi_{i}\right\} \\ \text { otherwise }\end{array}\right.$

Let $\left(K, \preceq_{K}\right)$ be the ordered base associated with $\Sigma . \quad K=\left\{\varphi_{i}:\left(\varphi_{i}, a_{i}\right) \in \Sigma\right\}$ and $\varphi_{i} \leq_{K} \varphi_{j}$ iff $a_{j} \leq a_{i}$, where $a_{i}$ and $a_{j}$ are the weights associated with $\varphi_{i}$ and $\varphi_{j}$ respectively.

Now defining, $\omega \leq_{\pi} \omega^{\prime}$ iff $\pi_{\Sigma}\left(\omega^{\prime}\right) \leq \pi_{\Sigma}(\omega)$, we can check that:

$$
\text { for } i=1,2: \omega \leq_{\mathcal{W}, i} \omega^{\prime} \text { iff } \omega \leq_{\pi} \omega^{\prime} \text {. }
$$

\section{Syntactic inference}

This section provides syntactic inference from $\left(K, \preceq_{K}\right)$. We first need to define from $\left(K, \preceq_{K}\right)$ a preference relation, $\preceq_{\mathcal{C}}$, between consistent subsets of $K$, denoted by $\mathcal{C}$. $C \preceq_{\mathcal{C}}$ $C^{\prime}$ means that the consistent subset $C$ is preferred to the consistent set $C^{\prime}$.

As it is said in section 2, in case of partial pre-orders, there are two possible ways to define $\preceq_{\mathcal{C}}$, depending if we use Definition 1 or Definition 2.

Definition 5 Let $C_{1}, C_{2} \in \mathcal{C}$. Then $C_{1} \preceq \mathcal{C}, i$ $C_{2}$ iff $\left\{\varphi_{2} \notin C_{2}\right\} \preceq_{i}\left\{\varphi_{1} \notin C_{1}\right\}$.

Intuitively, $C_{1}$ is preferred to $C_{2}$ if the preferred element outside $C_{1}$ is less preferred than the preferred element outside $C_{2}$.

We denote $C O N S_{i}$ the set of preferred consistent subset of $K$, with respect to $\preceq_{i}$. More formally:

Definition $6 C O N S_{i}=\operatorname{Min}\left(\mathcal{C}, \preceq_{\mathcal{C}, i}\right)$. 
Example 3 Let us consider again Example 2. $\mathcal{C}$ is composed by six consistent subsets, which are given by Table 1 .

Table 1: Set of consistent subsets of $K$.

\begin{tabular}{|c|c|c|}
\hline$C_{i}$ & $\left\{\varphi \in C_{i}\right\}$ & $\left\{\varphi \notin C_{i}\right\}$ \\
\hline \hline$C_{0}$ & $\{\emptyset\}$ & $\{a, b, \neg b\}$ \\
$C_{1}$ & $\{a\}$ & $\{b, \neg b\}$ \\
$C_{2}$ & $\{b\}$ & $\{a, \neg b\}$ \\
$C_{3}$ & $\{\neg b\}$ & $\{a, b\}$ \\
$C_{4}$ & $\{a, b\}$ & $\{\neg b\}$ \\
$C_{5}$ & $\{a, \neg b\}$ & $\{b\}$ \\
\hline
\end{tabular}

$C_{0}$ is the particular case where no formulas are considered from $K$.

According to the definition of $\preceq_{\mathcal{C}, 1}$, we obtain:

$$
\left\{\begin{array}{l}
C_{4} \preceq_{\mathcal{C}, 1} C_{1} \preceq_{\mathcal{C}, 1} C_{2}={ }_{\mathcal{C}, 1} C_{3} \preceq_{\mathcal{C}, 1} C_{0} \\
C_{5} \preceq_{\mathcal{C}, 1} C_{1}
\end{array}\right.
$$

For instance, let us show the inequality $C_{4} \preceq_{\mathcal{C}, 1} C_{1}$. Indeed the only formula which does not belong to $C_{4}, \neg b$, does not belong to $C_{1}$. We have then, by reflexivity of $\preceq_{K}$, $\neg b \preceq_{K} \neg b$.

Let us also show the inequality $C_{1} \preceq{ }_{\mathcal{C}, 1} C_{2}$. Two formulas are not in $C_{1}, b$ and $\neg b$. For each of these formulas, we can find a preferred element, which do not belong to $C_{2}$, namely $a$. Hence $C_{1} \preceq_{\mathcal{C}, 1} C_{2}$.

From $\preceq c, 1$ we get:

$$
\begin{aligned}
C O N S_{1} & =\operatorname{Min}(\mathcal{C}, \preceq \mathcal{C}, 1) \\
& =\left\{C_{4}, C_{5}\right\} \\
& =\{\{a, b\},\{a, \neg b\}\} .
\end{aligned}
$$

In this particular case, we have CON $S_{1}=$ $\mathrm{CONS}_{2}$.

The following gives the definition of syntactic inference.

Definition 7 A formula $\varphi$ is syntactically inferred from $\left(K, \preceq_{K}\right)$ (denoted $\left(K, \preceq_{K}\right) \vdash_{i}$ $\varphi)$ iff $\forall C \in C O N S_{i}, C \cup \neg \varphi$ is inconsistent.
Example 4 Let $\varphi=a \vee b$. We have $\neg \varphi=$ $\neg a \wedge \neg b$.

Recall that $C O N S_{i}=\{\{a, b\},\{a \neg b\}\} . W e$ have $\{a, b\}$ and $\{a, \neg b\}$ both inconsistent with $\neg \varphi$. Hence $\left(K, \preceq_{K}\right) \vdash_{i} \varphi$.

Note that this result is the same than the one found in Example 2, using the semantic inference.

Again the syntactic inference extends the syntactic inference of possibilistic logic, when we only deal with totally ordered knowledge. Namely, let $\Sigma$ be a possibilistic knowledge base. Recall that the syntactic possibilistic inference, denoted by $\vdash_{\pi}$, is obtained by only considering the most certain and consistent formulas with $\varphi$. See [3] for more details.

Then if we define $\left(K, \preceq_{K}\right)$ from $\Sigma$ as in section 4 , we can check:

$$
\left(K, \preceq_{K}\right) \vdash_{i} \varphi \text { iff } \Sigma \vdash_{\pi} \varphi \text { for any } \mathrm{i}=1,2 \text {. }
$$

Moreover, note that when $\preceq_{K}$ is a total preorder then $C O N S_{1}=C O N S_{2}$. Moreover, there exists an element $A$ in $C O N S_{i}$, such that for each $B \in C O N S_{1}$, we have $A \subseteq B$. Hence, for a total pre-order, the inference can be only based on one consistent subset of $K$, obtained by taking intersection of all elements in $C O N S_{i}$.

These syntactic inferences are equivalent to the semantic inferences. Namely, if we transform the partial pre-order on formulas $\preceq_{K}$ to a partial pre-order on interpretations, then applying the semantic inference using Definition 3 leads to the same result than applying syntactic inference using Definition 7. More formally:

Theorem 1 Let $\left(K, \preceq_{K}\right)$ be a partially ordered knowledge. Let $\preceq_{\mathcal{W}, i}$ be a partial preorder on interpretations obtained using Definition 4. Then:

$$
\left(K, \preceq_{K}\right) \vdash_{i} \varphi \text { iff }\left(\mathcal{W}, \preceq_{\mathcal{W}, i}\right) \models \varphi .
$$




\section{Conclusion and perspectives}

This paper has proposed an extension of possibilistic logic when dealing with partially pre-ordered knowledge.

Two definitions of semantic and syntactic inferences are provided. Both of them extends the one used in possibilistic logic. Moreover, the syntactic inference is sound and complete with respect to the proposed semantics.

A future work will be to develop algorithms for the two syntactic inferences. These algorithms will be applied in geographical information system where available knowledge are often partially ordered.

\section{Acknowledgements}

This work has been supported by the European project REV!GIS \#IST-1999-14189.

http://www.uni.univ-mrs.fr/REVIGIS

\section{References}

[1] S. Benferhat, D. Dubois, J. Lang, H. Prade, A. Saffiotti, and P. Smets. Reasoning under inconsistency based on implicitly-specified partial qualitative probability relations: a unified framework. In Proc. of 15th National Conferenc on Artificial Intelligence (AAAI-98), pages $121-126$.

[2] G. Brewka. Preferred subtheories: an extended logical framework for default reasoning. In Proc. of the 11th Inter. Joint Conf. on Artificial Intelligence (IJCAI'89), pages 1043-1048, 1989.

[3] D. Dubois, J. Lang, and H. Prade. Possibilisitc logic. In Handbook of Logic in Artificial Intelligence and Logic Programming, volume 3, pages 439-513. Oxford University Press, 1994.

[4] D. Dubois and H. Prade. Possibility Theory-An Approach to Computer- ized Processing of Uncertainty. Plenum Press, New York, 1988.

[5] J. Y. Halpern. Defining relative likelihood in partially-ordered preferential structures. Journal of AI Research, (7):1-24, 1997.

[6] J. Y. Halpern. Plausibility measures: A geeral approach for representing uncertainty. In Proceedings of the 17th International Joint Conference on AI (IJCAI 2001), pages 1474-1483, 2001.

[7] W. Spohn. Ordinal conditional functions : a dynamic theory of epistemic state. Causation in Decision, Belief Change and Statistics, pages 105-134, 1998. 